\title{
Verbal memory impairment after left insular cortex infarction
}

\author{
Facundo Manes, Jane Springer, Ricardo Jorge, Robert G Robinson
}

\begin{abstract}
PET studies have shown an association between changes in blood flow in the insular cortex and verbal memory. This study compared verbal memory profiles between a group of four right handed patients with right insular infarction and a group of six right handed patients with left insular infarction. Patient groups were comparable in age, education, and sex. Patients were administered memory tests about 4-8 weeks poststroke. Patients with left insular lesions showed significantly poorer immediate and delayed verbal memory as measured by story $A$ of the WMS-R logical memory I $(t=-2.73$, $p<0.03)$ and logical memory II $(t=-4.1$, p <0.004) subtests as well as the CERAD word list memory (delayed recall) $(t=-2.4$, $\mathrm{p}<0.05$ ). These findings indicate that left insular damage is associated with poorer performance on verbal memory tasks. The findings suggest that the insula may be part of a functional network that mediates verbal memory.

(F Neurol Neurosurg Psychiatry 1999;67:532-534)
\end{abstract}

Keywords: insula; memory; stroke

The insular cortex is reciprocally interconnected with all architectonic sectors of the adjacent lateral orbital and temporopolar regions. Many connections of the lateral orbital cortex with other non-paralimbic cortical areas are similar to those of the insula. ${ }^{1}$ Given the role of the left temporal lobe in verbal memory and, based on insular connections with this structure, it might be expected that lesions of the insular cortex would lead to verbal memory impairment. PET studies in normal volunteers have found that activation of the insula was associated with verbal memory tasks. ${ }^{23}$ In addition, insular cortex infarcts can produce aphasia $^{45}$ and memory complaints often persist despite good language recovery. ${ }^{6}$

In the present study we tested the hypothesis that damage to the left insular cortex would lead to a verbal memory impairment. Performances on verbal memory tasks were compared between a group of patients with left insular lesions and a group with right insular damage.
Patients and methods

Patients were recruited from Younkers Rehabilitation Hospital in Des Moines, Iowa where they had been admitted for treatment after an acute cerebral infarction. Two patients with left insular damage who developed aphasia after acute stroke were excluded to mitigate the confounding effects that aphasia would have on verbal memory test performance. All patients included in this study performed within normal limits on the Boston diagnostic aphasia examination, the controlled oral word association test (COWAT), the reduced version (30 items) of the Boston naming test, the block design subtest of the Weschler adult intelligence scale, the Benton visual retention test, and the mini mental state examination.

Patients were evaluated after obtaining informed consent between 4 and 8 weeks after acute stroke.

NEURORADIOLOGICAL EVALUATION

Patients were included if they had a single lesion demonstrated by CT or MRI that showed an ischaemic infarction restricted to the insula and adjacent white matter. Application of the above criteria yielded six right handed patients with left insula damage, (figure A) and four right handed patients with right insula lesions, (figure B).

\section{MEMORY ASSESSMENT}

Verbal memory was assessed using the wordlist memory test from the CERAD battery and the logical memory and verbal paired associates subtest of the Weschler memory scale (WMS-r). Visuospatial memory was assessed using the Benton visual retention test (BVRT).

\section{STATISTICAL ANALYSIS}

Student's $t$ tests were conducted on group means for all continuous variables. For discrete variables, $\chi^{2}$ tests were used. When expected cell sizes were prohibitively small $(<5)$, Fisher's exact test was used.

\section{Results}

BACKGROUND CHARACTERISTICS

Patients were white and men except one patient in each group, who were were women. The mean age for the left insular group was 69 (SD 5.5) years and it was 68 (SD 7.2) years for the right insular group. The mean years of 
A

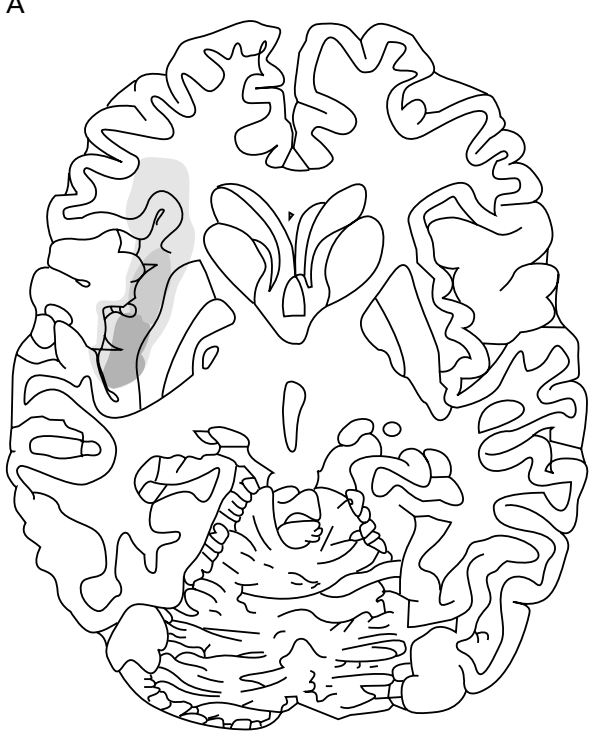

B

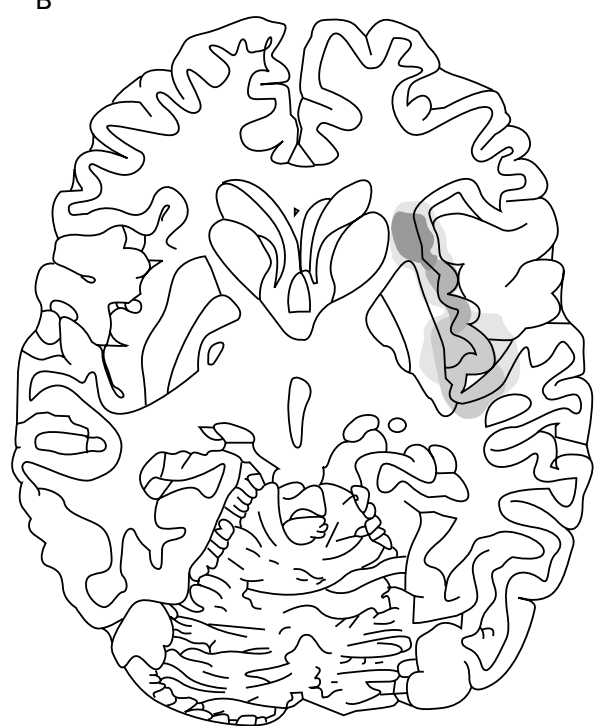

Figure(A) Schematic template showing the patients with left insular infarction. (B) Schematic template showing the patients with right insular infarction.

education for both groups was 11 . The mean time since stroke in the left insular group was 62 (SD 3.9) days and for the right insular group it was 59 (SD 3.6) days. The population was primarily composed of patients in Hollingshead classes III and IV. None of the patients had personal or family history of psychiatric disorder. The mean MMSE score for the right insular group was 27.8 (SD 1.1) and 27.7 (SD 1.1) for the left insular group. None of these intergroup differences were statistically significant.

\section{MEMORY FINDINGS}

Mean group scores did not differ significantly on measures of visual memory and visual perception (BVRT number correct, number of errors, table). Patients with left insular lesions showed significantly poorer verbal memory performances on the WMS-R logical memory I (story A) $(t=-2.73, \mathrm{df}=8, \mathrm{p}<0.03)$ and logical memory II (story A) $(t=-4.1, \mathrm{df}=8, \mathrm{p}<0.004)$ subtests. Poorer performance was also shown by the left group on the CERAD word list memory (delayed recall) $(t=-2.4, \mathrm{df}=6$, $\mathrm{p}<0.05$ ) (table).

Recall of verbal material, as reflected by performance on the logical memory story A subtest of WMS-R and the CERAD word list

Memory assessment

\begin{tabular}{lll}
\hline Test & $\begin{array}{l}\text { Left insula } \\
\text { Mean }(S D)\end{array}$ & $\begin{array}{l}\text { Right insula } \\
\text { Mean (SD) }\end{array}$ \\
\hline Logical memory I (story A) & $6.6(3.5)^{\star}$ & $12.2(2.5)$ \\
Logical memory II (story A 30 minute delayed recall) & $5(2.8)^{\star}$ & $11.8(1.8)$ \\
Visual paired associates I (total learning trials) & $16.8(4.4)$ & $15(3.5)$ \\
Visual paired associates II (total delay) & $4.2(2.8)$ & $4.4(2.6)$ \\
CERAD word list memory (trial I learning) & $4.8(2.2)$ & $3.3(2.0)$ \\
CERAD word list memory (trial II learning) & $5.6(1.5)$ & $6.6(2.3)$ \\
CERAD word list memory (trial III learning) & $6.6(0.8)$ & $7.6(0.5)$ \\
CERAD word list recall (delay) & $3.8(1.3)^{\star}$ & $6.9(1.0)$ \\
Savings & $57.6(15.7)^{\star}$ & $81.9(17.5)$ \\
BVRT(number correct) & $5.3(1.3)$ & $5.8(2.5)$ \\
BVRT(number of errors) & $9(5)$ & $9.6(4.8)$ \\
\hline
\end{tabular}

Values are means and SDs (presented in parentheses) for each of the memory and CERAD measures in the left and right insular damage groups. All comparations $p>0.5$. BVRT $=$ Benton visual retention test. ${ }^{\star} \mathrm{p}<0.005$. memory test, were also impaired in the left group in comparison with the normal range. The right insular damage group, however, performed within the normal range on all of these tests. ${ }^{78}$ Both groups performed within the normal range ${ }^{8}$ on tests of word list learning (table). The deficit shown by the left group involved primarily recall of verbal material compared with initial learning.

\section{Discussion}

The present study found that patients with left insular lesions had significantly poorer verbal memory than patients with right insular lesions. These findings implicate the left insular cortex in verbal memory circuits. They are consistent with PET studies in normal subjects, suggesting a role for the insular cortex in language and verbal memory tasks. ${ }^{23}$ This seems to be a selective deficit in verbal memory as non-verbal memory functions did not differ between groups. This dissociation of verbal and non-verbal memory test performance in our two patient groups should not be a surprise. Neuropsychological studies on surgery and non-surgery patients with temporal lobe epilepsy repeatedly showed a significant involvement of left temporolimbic structures in verbal memory processing. Studies examining the role of the right temporal lobe in figure or visual-spatial memory processing, however, have been less consistent. ${ }^{9} 10$

Before discussing the implications of these findings, it is acknowledged that only story A of the logical memory $\mathrm{A}$ and $\mathrm{B}$ subtests was administered, which is not in keeping with the standardised test. However, the right lesion patients performed within the normal range on the logical memory story A subtest ${ }^{7}$ suggesting that this change in test administration did not alter test performance. In addition, the group sizes were small and some of these findings need to be replicated in other patient groups. This is, however, the first study to perform 
group comparisons on the neuropsychological effects of lateralised insular strokes.

The cause of the impairment of verbal memory in patients with left insular lesions is of interest. Although the functional neuroanatomy of recall has not been determined, ${ }^{11}$ studies have shown verbal memory impairment in patients with left temporal lobe dysfunction. ${ }^{12} 13$ The insula sends efferents to the temporal cortex including the temporal pole and the supratemporal plane. Afferent fibres to the insula arise from the temporal lobe (including the temporal pole, primary auditory, auditory association, postauditory cortex, superior temporal cortex, and the temporal operculum). ${ }^{1}$ In addition, the insula may provide a neural relay for conveying sensory information into the limbic system. Connections between sensory areas and limbic structures are important because they provide a means for interrelating events in the external world with relevant motivational states. For this reason, Mesulam et al suggested that insular connections provide a fundamental anatomical substrate for learning and memory functions. ${ }^{14}$ On the basis of the above data and anatomical connections, ${ }^{15}$ the present findings might be construed as indicating that left insular lesions disrupt connections with areas that are necessary for executing memory tasks and therefore, lead to verbal memory deficits.

Moreover, Marshall et a $\bar{P}$ showed left temporal lobe hypoperfusion on SPECT in a patient who developed aphasia after left insular infarction. These authors suggested that hypoperfusion in the left temporal lobe may have reflected a functional disconnection between the posterior insula and language areas in the temporal lobe. The sudden removal of areas of functioning brain, as occurs with stroke, may produce distant effects in functionally connected neural structures. This phenomenon is often termed diaschisis. Thus, the verbal memory impairment in our left insular patients could be the result of diaschisis.
In conclusion, these findings indicate that left insular damage affects performance on certain verbal memory tasks to a greater extent than right insular damage. The findings also support the hypothesis that the insular cortex is part of a functional neuroanatomical circuit which mediates verbal memory. ${ }^{3}$

This work was supported by NIH grants MH 53592 and MH 52879 and has been presented in part at the 50th Annual Meeting Program of the American Academy of Neurology, ing Program of the American Academy of Neurology, Minneapolis, MN, USA, April 1998. We thank team members
of RGR's research laboratory for technical assistance. We also of RGR's research laboratory for technical assistance. We also
are grateful to Dr Diana Caine, who kindly made helpful suggestions on the preparation of the manuscript.

1 Mesulam M-M, Mufson EJ. Insula of the old world monkey, I: architectonics in the insulo-orbito-temporal componen of the paralimbic brain. F Comp Neurol 1982;212:1-22.

2 Grasby PM, Frith CD, Friston KJ, et al. A graded task approach to the functional mapping of brain areas implicated in auditory-verbal memory. Brain 1994;117: 1271-82.

3 Paulesu E, Frith CD, Frackowiak RSJ. The neural correlates of the verbal component of working memory. Nature 1993; 362:342-5.

4 Shuren J. Insula and aphasia. $\mathcal{F}$ Neurol 1993;240:216-18.

5 Marshall R, Lazar R, Mohr JP, et al. Semantic conduction aphasia from a posterior insular cortex infarction. $\mathcal{F}$ Neuroimaging 1996;6:189-91.

6 Risse GL, Rubens AB, Jordan LS. Disturbances of long-term memory in aphasic patients. A comparison of anterior and posterior lesions. Brain 1984;107:605-17.

7 Cauthen N. Extension of the Wechsler memory scale norms to the older age groups. F Clin Psychol 1977;33:208-12.

8 Welsh K, Butters N, Mohs R, et al. The Consortium to Establish a Registry for Alzheimer's Disease (CERAD). Establish a Registry for Alzheimer's Disease (CERAD).
Part V. A normative study of the neuropsychological Part V. A normative study of the
battery. Neurology 1994;44:609-14.

9 Mungas D, Ehlers C, Walton N, et al. Verbal learning differences in epileptic patients with left and right temporal lobe foci. Epilepsia 1985;26:340-5.

10 Jones-Gotman M, Smith M, Zatorre J. Neuropsychological testing for localizing and lateralizing the epileptogenic region. In: J Engel Jr, ed. Surgical treatment of the epilepsies. New York: Raven Press, 1993:245-62.

11 Cabezo R, Kapur S, Craik F, et al. Functional neuroanatomy of recall and recognition: a PET study of episodic memory. fournal of Cognative Neuroscience 1997;9:254-65.

12 Sass KJ, Buchanan BA, Kraemer BA, et al. Verbal memory impairment resulting from hippocampal neuron loss among epileptic patients with structural lesions. Neurology 1995:45:2154-8.

13 Sass KJ, Spencer DD, Kim JH, et al. Verbal memory impairment correlates with hippocampal pyramidal cell density. Neurology 1990;40:1694-7.

14 Mesulam M-M, Mufson E. Insula of the old world monkey. III: Efferent cortical output and comments on function. $\mathcal{F}$ Comp Neurol 1982;212:38-52. 